\title{
Divergent Molecular and Physiological Response of Two Maize Breeding Lines Under Phosphate Deficiency
}

\author{
Yanling Sun ${ }^{1} \cdot$ Hongxia Zheng ${ }^{2}$
}

Received: 11 June 2020 / Accepted: 8 August 2021 / Published online: 18 August 2021

(c) The Author(s) 2021

\begin{abstract}
Inorganic phosphate $(\mathrm{Pi})$ deficiency is a main limiting factor on crops growth and to select low-Pi tolerant breeding lines is very significant for crop breeding. Here, two contrasting maize (Zea mays L.) breeding lines showed different physiological response to Pi deficiency. The low-Pi tolerant QXN233 maintained normal growth, including high fresh weight, green leaves, strong shoots, and numerous roots relative to that of the sensitive MH05-4, mainly due to QXN233' high Pi content in shoots under Pi deficiency. Importantly, some Pi-responsive genes were detected, and among them, Pi transporters ZmPHT1;1 and ZmPHT1;9 as well as phytase gene Zmphytase 2 were expressed increasingly in QXN233 compared to MH05-4 under Pi deprivation or Pi resupply. Moreover, QXN233 had higher proline content, soluble sugar content, and SOD activity than MH05-4, related with its tolerance. Taken together, this study enriches the understanding of the mechanism of maize responding to Pi deficiency.
\end{abstract}

Keywords Maize $\cdot$ Pi deficiency $\cdot$ Tolerance $\cdot$ Marker genes $\cdot$ Breeding

\section{Introduction}

Phosphorus $(\mathrm{P})$ is an essential plant macronutrient, involved in the protein and nucleic acid biosynthesis, and energy metabolism, photosynthesis, respiration, the regulation of enzymes, and signal transduction cascades (Yang and Finnegan 2010). P is mostly at its bound or organic forms in soil for plants hardly to acquire, and thus plants usually

\section{Key Messages}

- The tolerant QXN233 has better growth and high Pi content in shoots relative to that of the sensitive MH05-4

- Pi transporters ZmPHT1;1 and ZmPHT1;9 as well as phytase gene Zmphytase 2 could be important regulators for the tolerance to Pi deficiency

Yanling Sun

sylsk046@163.com

Hongxia Zheng

zhenghongxia2020@foxmail.com

1 Marine Science and Engineering College, Qingdao Agricultural University, No. 17 Wenhai Road, Shandong 266237 Qingdao, China

2 Key Laboratory of Saline-alkali Vegetation Ecology Restoration (Northeast Forestry University), Ministry of Education, College of Life Sciences, Northeast Forestry University, No. 26 Hexing Road, 150040 Harbin, People's Republic of China exposed to Pi deficiency (Schachtman et al. 1998; Chiou and Lin 2011). Inorganic phosphate (Pi) has become one of the most limiting factors for plants growth and crop productivity. Plants have a variety of adaptive strategies to survive in $\mathrm{Pi}$ deficiency, including many physiological and biochemical responses to remobilize and optimize metabolic process to acquire more Pi into plant (Rausch and Bucher 2002; LópezArredondo et al. 2014; Sawers et al. 2017; Wang et al. 2017). However, the underlying mechanism of plants response to $\mathrm{Pi}$ deficiency is not clearly clarified (Chiou and Lin 2011; Liu et al. 2016; Ham et al. 2018).

Plant roots is directly sensing local Pi levels and influenced by Pi deficiency, including the inhibition of primary root (PR) growth and the promotion of lateral root formation (Vance et al. 2003). Recent studies showed that Pi deficiency induces plant to accumulate high level of iron $(\mathrm{Fe})$ in the apoplast of roots, resulting in the production of reactive oxygen species (ROS) and the increase of callose deposition in plasmodesmata, which interferes with the intercellular movement of SHORT ROOT (SHR) protein and causes the impairment of PR growth (Müller et al. 2015; Wang et al. 2019). Besides root level modifications, aerial parts of plants also show key phenotypic modifications (Ruan et al. 2018; Ceasar et al. 2020). Responding to Pi deficiency, plants adapted themselves to optimize Pi acquisition and 
redistribute Pi into the young or starved tissues under the control of the Pi uptake and regulatory pathway (Rausch and Bucher 2002; Chiou and Lin 2011; Liang et al. 2014; Mitra 2015; Ham et al. 2018). Among them, a MYB-like transcription factor phosphate starvation response 1 (PHR1) protein plays central role, which is a key molecular to regulate the expression of a large number of $\mathrm{P}$ starvation-responsive genes, such as many Pi transporters (PHTs), induced by phosphate starvation 1 (IPS1), RNS1 (a RNase), and SPX genes by binding to their promoters through the cis-element PHR1-binding sequence (P1BS; GNATATNC) (Rubio et al. 2001; Calderón-Vázquez et al. 2011; Liu et al. 2018; Xu et al. 2018). SPX (named after SYG1, PHO81, and Xpr1) families were classified into four families based on the SPX, SPX-EXS, SPX-MFS, and SPX-RING domain for each, and involved in Pi homeostasis via binding different inositol polyphosphate signalling molecules (InsPs) with several other proteins, including PHRs (Secco et al. 2012; Liu et al. 2018; Ried et al. 2021). IPS1 antagonizes miR-399 activity, and miR-399 can further guide the cleavage of PHO2 RNA, which encodes an E2 ubiquitin conjugase-related protein that negatively affects shoot Pi content (Franco-Zorrilla et al. 2007). Thus, PHO2 mutations masked the effects of IPS1 overexpression on Pi accumulation in shoots (FrancoZorrilla et al. 2007). IPS1 was found to be induced by low-Pi (LP) stress and hormone treatment (Franco-Zorrilla et al. 2007; Zhang et al. 2019). The PHO1 family members, containing both the SPX and EXS domains, involved in Pi loading into the xylem in the long-distance Pi deficiency signalling network (Wang et al. 2004; Stefanovic et al. 2007; Secco et al. 2012). Importantly, PHT1 4 families were taken part in Pi uptake in plants (Poirier and Bucher 2002; Lin et al. 2009; Liu et al. 2016; Sawers et al. 2017; Wang et al. 2017). Previously, AtPHT1;1 and AtPHT1;4 were remarkably enhanced in Arabidopsis roots in response to Pi-starvation. Overexpression of $P H T 1 ; 1$ improved Pi uptake to promote the cell growth in tobacco (Mitsukawa et al. 1997; Lin et al. 2009), and knocking out of PHT1;1 or PHT1;4 impeded Pi uptake (Misson et al. 2004; Shin et al. 2004). In maize (Zea mays L.), the ZmPHT1 families had been identified to be contributed to maintain Pi uptake and regulate Pi homeostasis (Nagy et al. 2006; Gu et al. 2016; Liu et al. 2016; Wang et al. 2017). Moreover, phytate-P, which makes up $50 \%$ component of the total soil organic $\mathrm{P}$, is poorly utilized by the roots of most plants, and phytases are a special class of phosphatase that hydrolyzes phytate to release inorganic Pi. Phytase-overexpressing transgenic maize could improve Pi availability (Chen et al. 2008).

Under different abiotic stresses, compatible osmoprotectants, such as proline, glycine betaine, trehalose, and polyols, are accumulated to protect plants from various abiotic stresses including Pi deficiency (Nuccio et al. 1999; Vinocur and Altman 2005). Under stresses, proline was highly increased and helpful to maintain osmosis balance, stabilize protein structure, and scavenge excess reactive oxygen species (ROS), associated with the increased/decreased expression of proline synthetases/dehydrogenase (Verslues and Sharma 2010; Spoljarević et al. 2011; Chen et al. 2013; Rai and Penna 2013; Sun et al. 2015). Recent research supported that proline content was positively correlated with maize crop yields (Spoljarević et al. 2011). Moreover, trehalose, as a non-reducing disaccharide, could be necessary for cellular membrane integrity to maintain osmotic balance in plants cell. It was all be accumulated among various plant species to resist with extreme conditions, and overexpression of trehalose-6-phosphate synthase (TPS1) gene could improve the tolerance of plants under stresses (Elbein et al. 2003; Avonce et al. 2005).

In recent years, plenty of Pi-responsive genes had been identified using high throughput sequencing technology and provided valuable information to understand the mechanism of plants adapt to Pi deficiency (Calderon-Vazquez et al. 2008; Lin et al. 2013; Oono et al. 2013; Pei et al. 2013; Sun et al. 2016a, b; Jiang et al. 2017). Here, two contrasting maize inbred lines, QXN233 and MH05-4, exhibited different physiological response under Pi deficiency, and this study tried to reveal the underlying mechanism of them during Pi deficiency.

\section{Materials and Methods}

\section{Plant Growth and Treatments}

QXN233 and MH05-4, as stable maize inbred lines, derived from Reid and Heterotic Pattern B (PB) inbred lines, respectively. Experiments were carried out in the artificial culture chamber at Shandong Academy of Agricultural Sciences/ National Engineering Laboratory of Wheat and Maize. The light/dark time was $14 / 10 \mathrm{~h}$ and the temperature and relative humidity controlled at $26 \pm 4{ }^{\circ} \mathrm{C}$ and $60-65 \%$, respectively. The seeds were surface-sterilized by $10 \% \mathrm{NaClO}$ for $10 \mathrm{~min}$, germinated in a chamber at $28^{\circ} \mathrm{C}$, and then grown in the normal liquid Hoagland's nutrient solution for a week, its composition was as follows: $25 \mathrm{mM} \mathrm{H}_{3} \mathrm{BO}_{4}, 2 \mathrm{mM} \mathrm{Ca}\left(\mathrm{NO}_{3}\right)_{2}, 0.65 \mathrm{mM} \mathrm{MgSO}_{4}$, $0.5 \mathrm{mM} \mathrm{KH}_{2} \mathrm{PO}_{4}, 50 \mu \mathrm{M} \mathrm{KCl}, 25 \mu \mathrm{M}$ Fe-EDTA, $5 \mu \mathrm{M} \mathrm{MnSO}_{4}$, $2 \mu \mathrm{M} \mathrm{ZnSO}_{4}, 0.5 \mu \mathrm{M} \mathrm{CuSO}_{4}$, and $0.005 \mu \mathrm{M}\left(\mathrm{NH}_{4}\right)_{6} \mathrm{Mo}_{7} \mathrm{O}_{24}$. The fresh nutrient solution was replaced every day. Seven-dayold seedlings were treated with the low-Pi $(\mathrm{LP})$ stress $(0 \mathrm{mM} \mathrm{Pi}$, $\mathrm{K}^{+}$was supplied by the equal concentration of $\mathrm{KCl}$ rather than $\left.\mathrm{KH}_{2} \mathrm{PO}_{4}\right)$ and optimum $\mathrm{Pi}\left(500 \mu \mathrm{M} \mathrm{KH}_{2} \mathrm{PO}_{4}\right.$, control) solution for 0 day, 2 days, 14 days, or 45 days. In addition, after treated for 14 days under Pi deficiency, seedlings were then applied to the normal Hoagland's nutrient solution for 2 days as a Pi resupply. The $0.5 \mathrm{~L}$ solutions were applied newly every day. 
For the quartz sand or soil analyses of pot, the seedlings at the three-leaf stage were treated with the LP solution for 20 days. The same $0.5 \mathrm{~L}$ fresh solutions as the above were applied every 2 days. The plant height, leaf width, leaf length, and fresh weight $(\mathrm{FW})$ of leaves or roots were weighed by chemical balance and analyzed between each groups.

\section{Measurements of MDA and Anthocyanin Contents}

Seven-day-old seedlings were treated under Pi deficiency for 2 days, 14 days, or 45 days, respectively. MDA and anthocyanin contents were measured following the previous methods (Sunarpi et al. 2005; Sun et al. 2016a, b).

\section{Measurements of Pi, Proline, Soluble Sugar, and Soluble Protein Content}

The shoots were harvested from the different treated time points, and Pi content was determined following previous method (Sun et al. 2016a, b). Meanwhile, the leave samples were collected after treatment. The proline, soluble sugar, and soluble protein contents were analyzed, respectively, following as the previous protocols (Sun et al. 2015).

\section{SOD Activity Measurement}

Seven-day-old seedlings were treated for 2 days using a hydroponic assay under Pi deficiency and the leave samples were collected after treatment. Then, the leaves SOD activity from the Pi deficiency and control group was measured in accordance with the previously described protocols (Sun et al. 2015).

\section{Quantitative Real-Time PCR (qRT-PCR) Analysis}

Seven-day-old seedlings were treated for 14 days of Pi deficiency and 2 days of Pi resupply, respectively. Total RNA were extracted from the treated leave and root samples using the E-Z Nucleic Acid (E.Z.N.A) Plant RNA Kit (Cat.nos. R6827-01, OMEGA), and cDNA was synthesized by using $5 \times$ All-in-One RT MasterMix Kit (ABM, Canada). qRT-PCR was then performed using Ultra SYBR Mixture Kit on ABI 7500 Real-Time PCR System (ABI, USA) (low ROX, CW2601M, CWBIO), following the manufacturer's instructions: $95^{\circ} \mathrm{C}$ for $2 \mathrm{~min}, 40$ cycles at $95{ }^{\circ} \mathrm{C}$ for $15 \mathrm{~s}$, and $60{ }^{\circ} \mathrm{C}$ for $1 \mathrm{~min}$. The primers for qRT-PCR were presented in Table 1. Using $18 \mathrm{~S}$ rRNA as the endogenous control, each sample was analyzed thrice, and the $2^{-\Delta \Delta \mathrm{Ct}}$ method was calculated the relative transcript abundance of the target gene (Schmittgen and Livak 2008).
Table 1 Primers used in qRTPCR

\begin{tabular}{|c|c|c|}
\hline Gene ID & Gene name & Sequences $\left(5^{\prime}-3^{\prime}\right)$ \\
\hline \multirow[t]{2}{*}{ AF168884 } & 18SrRNA-F & CCATCCCTCCGTAGTTAGCTTCT \\
\hline & $18 S r R N A-\mathrm{R}$ & CCTGTCGGCCAAGGCTATATAC \\
\hline \multirow[t]{2}{*}{ GRMZM2G326707 } & ZmPHT1;1-F & TCGTCACGCTCGTCATCTCC \\
\hline & ZmPHT1;1-R & GGCATCTTCATCCGCCAGTAG \\
\hline \multirow[t]{2}{*}{ GRMZM2G154090 } & ZmPHT1;9-F & CAACCAGGACAGGAGCAAGAC \\
\hline & ZmPHT1;9-R & TGACGGGCTCCTCCTCTGA \\
\hline \multirow[t]{2}{*}{ GRMZM2G043336 } & Zmphytase 2-F & CGGGCAACCTGGCGTGG \\
\hline & Zmphytase 2-R & CGCCTTGGTGACAGCCGC \\
\hline \multirow[t]{2}{*}{ GRMZM2G006477 } & ZmPHRI-F & TGGTGGGCAGATATAATGAATGATG \\
\hline & ZmPHRI-R & CTATTGGGAGGACTAGCAACAGGAC \\
\hline \multirow[t]{2}{*}{ Zm00001d042935 } & ZmIPS1-F & GTCCTCATCCAACACCTTCC \\
\hline & ZmIPS1-R & CACACGCACTCCATTACCAT \\
\hline \multirow[t]{2}{*}{ GRMZM2G171423 } & $\mathrm{ZmSPX1- \textrm {F }}$ & TTCGCATCACCAGCACCACC \\
\hline & $\mathrm{ZmSPX1- \textrm {R }}$ & CGAACTTCATTGTTGCGATTGG \\
\hline \multirow[t]{2}{*}{ GRMZM2G466545 } & $\mathrm{ZmPHO1-F}$ & GCAGCAGCCCAGGAACACC \\
\hline & $\mathrm{ZmPHO1-R}$ & GATGGACGCCGACAAGAGGA \\
\hline \multirow[t]{2}{*}{ DQ026301 } & $\mathrm{ZmP5CR}-\mathrm{F}$ & CCAGCCTGTGCCAACCGC \\
\hline & $\mathrm{ZmP5CR}-\mathrm{R}$ & GTGCGGATGGCGGAGGC \\
\hline \multirow[t]{2}{*}{ DQ864376 } & $\mathrm{ZmP5CS}-\mathrm{F}$ & GCGAGGAAGTGGGCAAGTGGT \\
\hline & ZmP5CS-R & TTGGGGAGGTGGGGTGGC \\
\hline \multirow[t]{2}{*}{ AF529266 } & ZmTPS1-F & GGTTGCAGCGTTTCCTATTG \\
\hline & ZmTPS1-R & AATCAAGAGATCGGTCCAGATG \\
\hline \multirow[t]{2}{*}{ GRMZM2G169890 } & ZmSOD4-F & TAAGCACCTGTGGCAACCGAT \\
\hline & ZmSOD4-R & ACGAAACGGTCGGAATGCC \\
\hline \multirow[t]{2}{*}{ GRMZM2G427815 } & $\mathrm{ZmPOD3- \textrm {F }}$ & TGACTGCTTTGTCCAGGGGTG \\
\hline & ZmPOD3-R & GACTGCCTCCACCTGTGCCTT \\
\hline
\end{tabular}




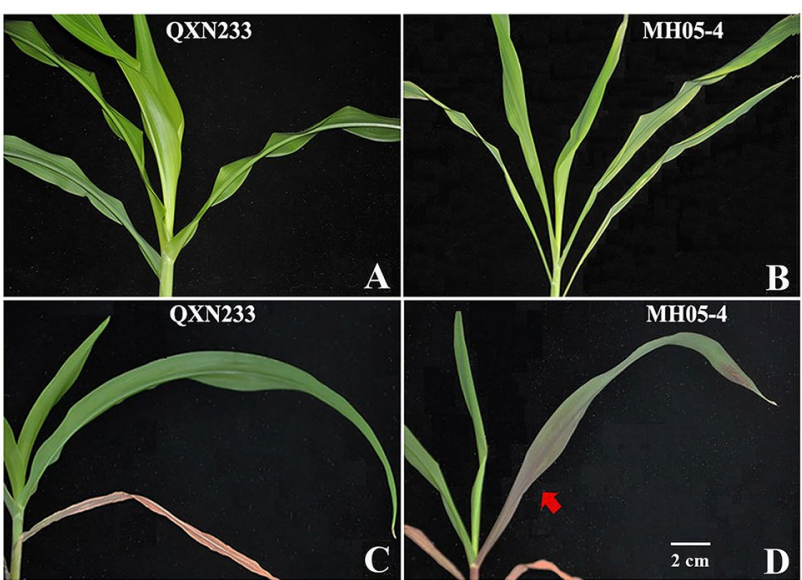

$\mathbf{E}$

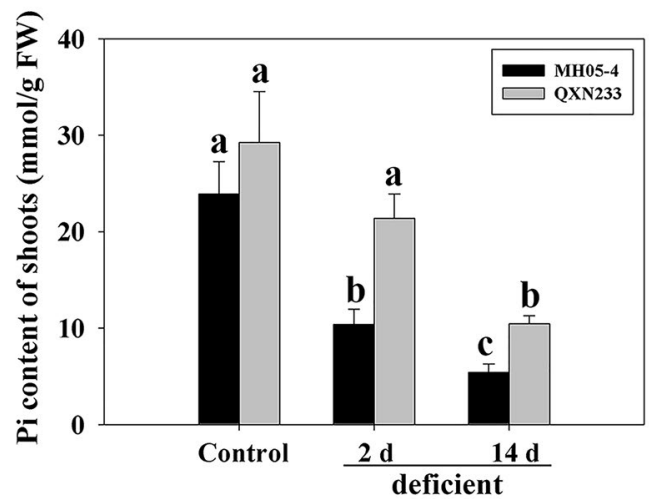

Fig. 1 The leaves phenotypic responses and Pi content of two contrasting maize breeding lines to $\mathrm{Pi}$ deficiency. The phenotype of QXN233 (A) and MH05-4 (B) under control condition and phenotype of QXN233 (C) and MH05-4 (D) under Pi deficiency for 45 days. The red arrow showed the red-purple leaves in MH05-4. Bar $=2 \mathrm{~cm}$; Pi content of shoots in QXN233 and MH05-4 (E). Maize seedlings were grown under Pi deficiency for 2 days and 14 days. The lowercase indicates significantly different $(P<0.05, n=3$ replicates. 5-16 seedlings were tested for each genotype)

\section{Statistical Analysis}

The data were shown as average values \pm standard deviations (SD) from three or five independent repeats. All data obtained were subjected to one-way analysis of ANOVA by Duncan's test with SPSS (17.0) software (IBM, USA), and the significant differences were defined at the $P$ value $(* P<0.05)$.

\section{Results}

\section{Characteristics of Two Contrasting Breeding Lines Under Pi Deficiency Stress}

In the present study, several maize inbred lines were used to screen their tolerance under Pi deficiency based on the physiological LP-tolerance indexes. As a result, QXN233 kept the highest Pi content and superior performance, selected as the tolerant line, whereas MH05-4 showed the lowest Pi content and highest leaf anthocyanin content as the sensitive one, similarly to the sensitive QXH0121 in our previous study (Figs. 1 and 2; Table 2). In order to deplete endogenous Pi rapidly and exhibit a Pi deficiency symptom as soon as possible, $0 \mu \mathrm{M} \mathrm{KH_{2 }} \mathrm{PO}_{4}$ condition was used as Pi deficiency for three-leaf-stage seedlings to suffer, while optimum $\mathrm{Pi}\left(500 \mu \mathrm{M} \mathrm{K \textrm {K } _ { 2 }} \mathrm{PO}_{4}\right)$ condition as a control. The results showed that QXN233 and MH05-4 both had weakly growth under Pi deficiency compared to normal conditions (Table 3). Obviously, MH05-4 seedlings exhibited an obvious red-purple in leaves, as a typical Pi-deficient symptom whereas the leaves of QXN233 seedlings remained green (Figs. 1, 2, S1 and S2). MH05-4 accumulated more MDA (Fig. 2A), and had smaller number of roots and lower fresh weight than QXN233 did under Pi deficiency (Fig. 2B-D; Table 3).

Significantly, Pi content of QXN233 has over twice than MH05-4 in shoots (Fig. 1E), indicating that an enhanced shoot $\mathrm{Pi}$ accumulation contributed to the tolerance of QXN233. Furthermore, the differences accumulation of compatible solutes between QXN233 and MH05-4 were investigated. The results showed that proline contents were both increased in two tested breeding lines under Pi deficiency, and QXN233 has higher proline content than that of MH05-4 (Fig. 3A); the same response of soluble sugar emerged between QXN233 and MH05-4 (Fig. 3B). Besides, QXN233 had slightly high soluble protein content than that of MH05-4 (Fig. 3C). In addition, SOD activity was higher in QXN233 than in MH05-4 under Pi deficiency (Fig. 3D).

\section{Expression of Pi-Responsive Genes in Two Contrasting Breeding Lines}

To test whether some important Pi-responsive genes were differently expressed between QXN233 and MH05-4 under Pi deficiency, the upstream regulators ZmPHRI (GRMZM2G006477), ZmIPSI (Zm00001d042935), ZmSPXI (GRMZM2G171423), and ZmPHOI (GRMZM2G466545), as well as Pi transporters ZmPHT1;1 (GRMZM2G326707) and ZmPHT1;9 (GRMZM2G154090), were examined by qRT-PCR. Under Pi deficiency, the tolerant QXN233 showed high expression levels of ZmPHR1, ZmIPS1, ZmSPX1, and $\mathrm{ZmPHO1}$ relative to that of MH05-4 (Fig. 4). For Pi transporters, ZmPHT1;1 and ZmPHT1;9 were both upregulated in QXN233 than MH05-4 under Pi deficiency (Fig. 5A-D), consistent with its high Pi content in shoots (Fig. 1E). Especially for ZmPHT1;1 and ZmPHT1;9, were both increasingly in QXN233 leaves and roots after 14 days of Pi deficiency and subsequently 2 days of recovery, and enhanced by sevenfold and ninefold in QXN233 roots at 2 days of recovery, 
Fig. 2 Changes in MDA and anthocyanin contents of leaves, the length of primary roots, and the number of total roots in QXN233 and MH05-4 under Pi deficiency. A MDA contents; $\mathbf{B}$ anthocyanin contents; $\mathbf{C}$ the length of primary roots; and D the number of total roots. Maize seedlings were treated for 14 days under Pi deficiency. The lowercase indicates significantly different $(P<0.05, n=3$ replicates. 10-12 seedlings were tested for each genotype)
A

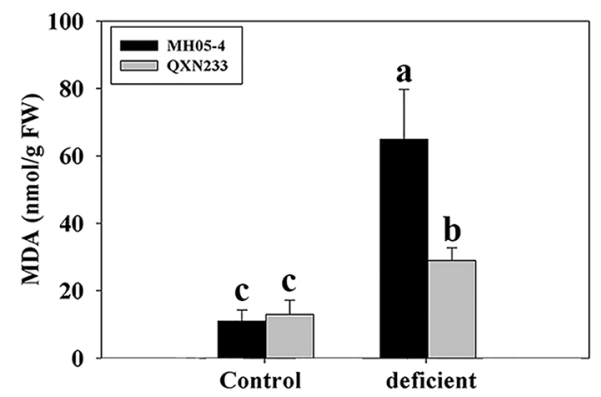

C

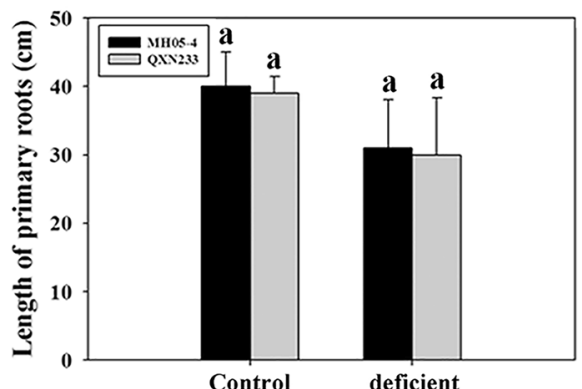

B

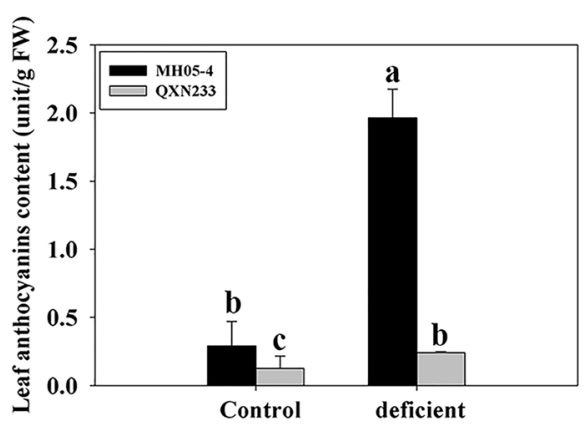

D

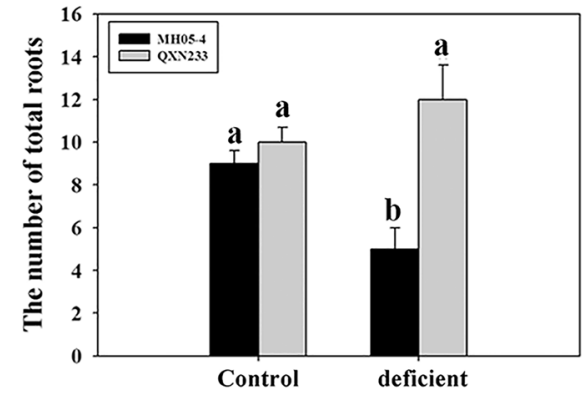

respectively (Fig. 5B and D). Also, MH05-4 has high expression levels of $Z m P H T 1 ; 1$ and $Z m P H T 1 ; 9$ in leaves or roots only at 2 days of recovery, and the degree of rise was low compared with that of QXN233 (Fig. 5A-D). Consistently, similar expressed pattern was also detected between QXN233 and the other sensitive QXH0121 (Fig. S4), previously reported in our study. These results suggested a stronger ability of Pi transport in tolerant QXN233 than the sensitive ones. Moreover, Zmphytase 2 was elevated more in QXN233 than in MH05-4 under Pi deficiency or recovery (Fig. 5E and F). Especially in roots, the transcript level of Zmphytase 2 was increased in QXN233, and had more than 2- and fourfold relative to that of MH05-4, suggesting that QXN233 has more phytase to catalyze phytate-P into available Pi, corresponding to its high Pi content (Fig. 1E). Also, a similar trend appeared between QXN233 and the sensitive QXH0121 (Fig. S4).

\section{Expression of Stress-Responsive Genes in Two Contrasting Breeding Lines}

Two proline-biosynthesis genes ZmP5CR and ZmP5CS were highly transcribed in QXN233 compared to MH05-4 (Fig. 6A and B), consistent with its higher proline content under Pi deficiency (Fig. 1E; Table 2). Moreover, QXN233 had increased expression of ZmTPS1, contributed to its high soluble sugar content under Pi deficiency (Figs. 3B and $6 \mathrm{C}$ ). In addition, consistent with the high SOD activity in QXN233, the transcription levels of ZmSOD4 and ZmPOD3 were both enhanced 50- or 60-fold compared to that of MH05-4 (Figs. 3D and 6D-E), hinting that QXN233 possessed high SOD and POD activity to reduce oxidative damage and lipid peroxidation under LP stress.

\section{Discussion}

As one of common abiotic stresses, Pi deficiency severely impeded the plant growth and production in maize. To understand the mechanism of maize response to Pi deficiency, selecting tolerant materials are vital for breeding and crop yield. In our study, two maize breeding lines (QXN233 and MH05-4) were investigated to be exhibited contrasting responses under Pi deficiency. Obviously, QXN233 displayed a tolerant phenotype, including high fresh weight, strong shoots, and roots. Contrastingly,

Table 2 The measurements of anthocyanin and shoot Pi contents in eight tested maize genotypes. Maize seedlings were grown for 20 days under Pi deficiency using a quartz sand assay. Data were shown as means $\pm \mathrm{SD}(* P<0.05)$

\begin{tabular}{lll}
\hline Maize inbred lines & $\begin{array}{l}\text { Shoot Pi content } \\
(\mathrm{mmol} / \mathrm{g} \mathrm{FW})\end{array}$ & $\begin{array}{l}\text { Leaf anthocyanin } \\
\text { content (unit/g FW) }\end{array}$ \\
\hline MH05-4 & $18.9 \pm 1.5$ & $5.2 \pm 0.3$ \\
Xianyu335 & $20.5 \pm 5.8$ & $0.7 \pm 0.3$ \\
Qi319 & $21.4 \pm 4.5$ & $5.0 \pm 1.0$ \\
Chang7-2 & $22.5 \pm 5.3$ & $4.4 \pm 0.2$ \\
MH9311 & $24.0 \pm 6.0$ & $0.7 \pm 0.4$ \\
Zheng58 & $28.3 \pm 7.0$ & $1.2 \pm 0.6$ \\
MH03-2 & $28.7 \pm 5.6$ & $3.2 \pm 0.1$ \\
QXN233 & $31.0 \pm 6.7^{*}$ & $0.6 \pm 0.1^{*}$ \\
\hline
\end{tabular}


Table 3 Quantitative analyses of plant height, leaf width, and length of the longest leaf and fresh weights of leaves and roots of QXN233 or MH05-4 under Pi deficiency after 20 days, as assessed via a quartz sand pot experiment. The values represent the means \pm SD $(* P<0.05)$

\begin{tabular}{lllllcc}
\hline Genotype & Treatment & Height $(\mathrm{cm})$ & Leaf width $(\mathrm{cm})$ & Leaf length $(\mathrm{cm})$ & $\begin{array}{l}\text { Fresh weight of } \\
\text { leaves }(\mathrm{g})\end{array}$ & $\begin{array}{l}\text { Fresh weight } \\
\text { of roots }(\mathrm{g})\end{array}$ \\
\hline QXN233 & $0 \mathrm{mM} \mathrm{Pi}$ & $17.6 \pm 4.3$ & $2.4 \pm 0.3$ & $23.5 \pm 3.0$ & $2.8 \pm 0.1$ & $2.3 \pm 0.4$ \\
& Control & $33.2 \pm 6.1$ & $3.2 \pm 0.1$ & $33.3 \pm 4.1$ & $7.6 \pm 0.5$ & $5.4 \pm 0.3$ \\
MH05-4 & $0 \mathrm{mM} \mathrm{Pi}$ & $18.1 \pm 2.3$ & $1.8 \pm 0.2^{*}$ & $21.6 \pm 4.2$ & $2.0 \pm 0.2^{*}$ & $1.5 \pm 0.3^{*}$ \\
& Control & $34.5 \pm 1.2$ & $3.1 \pm 0.3$ & $33.4 \pm 3.2$ & $7.2 \pm 0.4$ & $5.2 \pm 0.2$ \\
\hline
\end{tabular}

MH05-4 exhibited weak performance and emerged purple leaves, as a typical symptom, and possessed high anthocyanin and MDA content, indicated that a high lipid peroxidation and ROS damage related with its inferior phenotype.

Previously reported, plants have adopted critical mechanisms to improve Pi acquisition and use efficiency under Pi deficiency (Poirier and Bucher 2002; Wang et al. 2017; Sawers et al. 2017). Many Pi-responsive genes, including Pi transporters and phytases, participated actively in Pi acquisition, transport, and homeostasis (Mudge et al. 2002; Nagy et al. 2006; Liu et al. 2016; Jiang et al. 2017; Wang et al. 2017; Ham et al. 2018). In the present study, the tolerant QXN233 showed twice high shoot Pi contents compared to MH05-4 under Pi deficiency, indicated that optimizing $\mathrm{Pi}$ allocation into shoot in maize probably as an important strategy to confront with Pi deficiency, which related with the roles of some Pi transporters. Among them, ZmPHT1; 1, ZmPHT1;9, and Zmphytase 2 were both enhanced obviously in QXN233 compared to that of the sensitive MH05-4 and QXH0121 under Pi deficiency or recovery, implying a vital role of them for Pi uptake and transport. Consistently, in our previous study, the RNA-Seq analysis showed that five maize inorganic Pi transporter genes, containing ZmPHT1; l, were highly expressed in the tolerant QXN233 (Sun et al. 2016a, b), associated with its tolerance. Thus, the Pi deficiency responsive genes are selected on the basis of their differential expression in tolerant and sensitive varieties based during Pi-deficient conditions. Nevertheless, they do not perfectly correlate with the Pi content, and possibly, these two genotypes have differential Pi requirements. Taken together, these results hinted us that ZmPHT1;1, ZmPHT1;9, and Zmphytase 2 could be vital genes for the LP tolerance of maize, which could be checked in a multitude of breeding lines under Pi deficiency via GWAS (genome-wide association study). Moreover, PHT1;1 was reported to take part in xylem loading process of $\mathrm{Pi}$ in Arabidopsis (Fang et al. 2009; Lin et al. 2009). The biological functions of these Pi transporters could be confirmed via the single or combined overexpression or RNAi of them in plants. Significantly, the initial level of these transcripts is lower in the leaves of the tolerant QXN233 than the susceptible MH05-4, indicated
Fig. 3 Changes in compatible solutes contents and SOD activity in QXN233 and MH05-4 leaves. A Proline, B soluble sugar, $\mathbf{C}$ soluble protein contents, and D SOD activity in MH05-4 and QXN233 were measured after seedlings of them treated for 2 days under Pi deficiency. The lowercase indicates significantly different $(P<0.05, n=3$ replicates. $5-10$ seedlings were tested for each genotype)
A

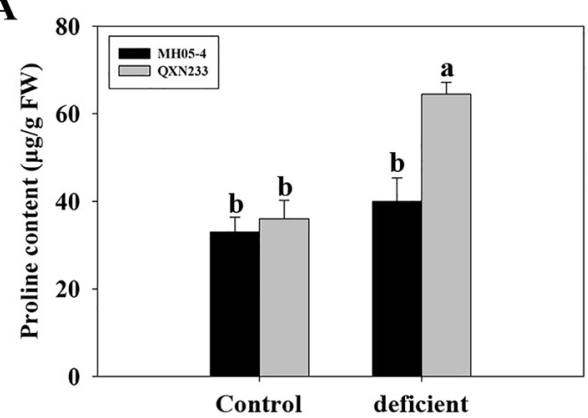

C

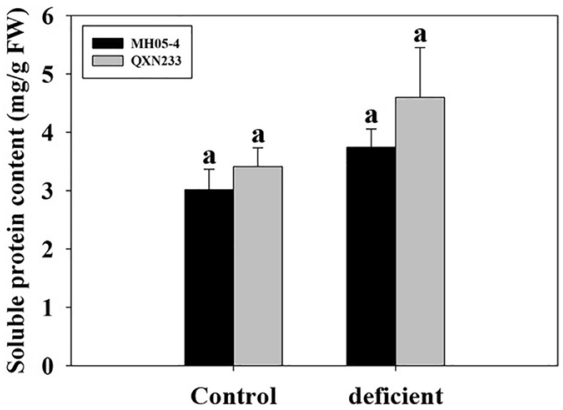

B

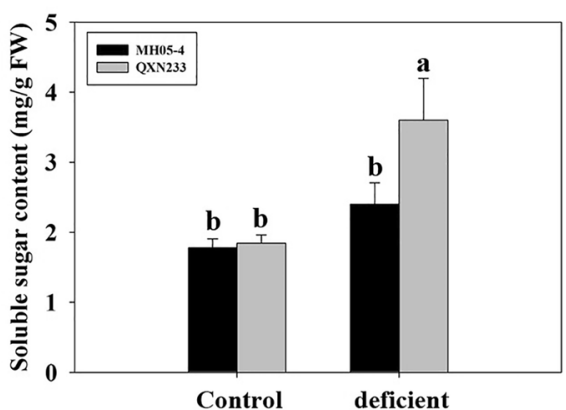

D

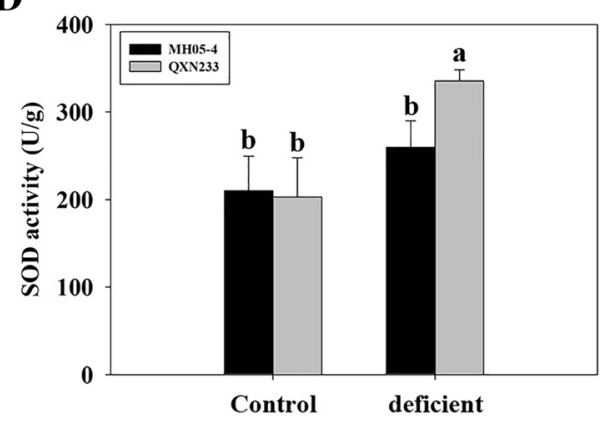


Fig. 4 Expression analysis of the upstream molecules of Pi signalling in QXN233 and MH05-4 by qRT-PCR. The transcript levels of $Z m P H R 1$ (A), ZmIPS1 (B), ZmSPX1 (C), and $\mathrm{ZmPHOl}$ (D) in leaves. Maize seedlings were treated for 2 days under Pi deficiency. Values given as mean \pm SD $(P<0.05, n=5$ biological replicates)
A

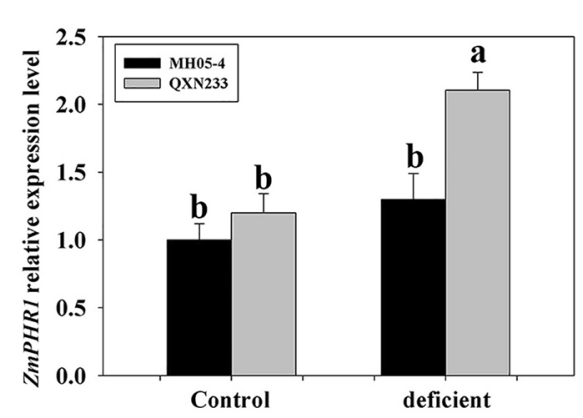

C

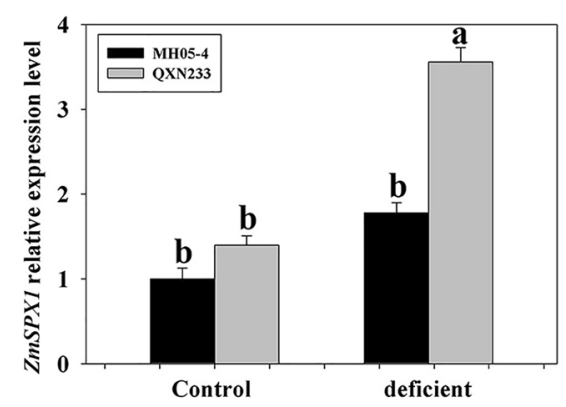

B

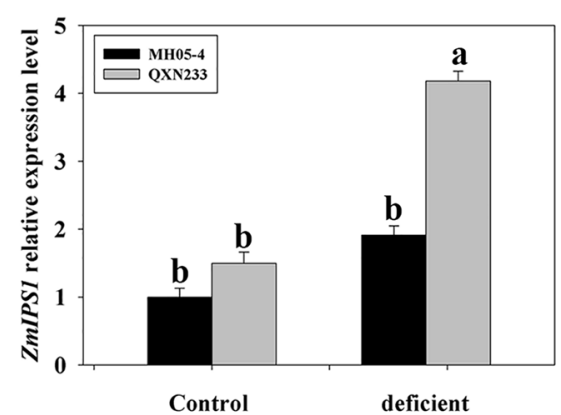

D

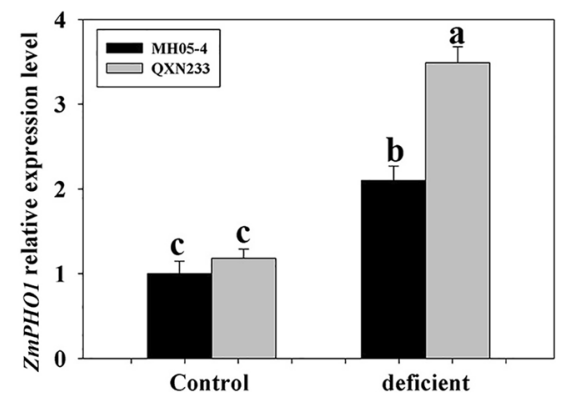

that not only the PHTs but also some upstream regulating factors of them are critical for the maintenance of plant LP tolerance. Correspondingly, the upstream regulators, such as ZmPHR1, ZmIPS1, ZmSPX1, and ZmPHO1, were detected to be upregulated in the tolerant QXN233 relative to that of the sensitive MH05-4. In addition, vacuolar phosphate efflux transporters were identified to be responsible for Pi release from the vacuoles to the cytoplasm in Oryza sativa (Xu et al. 2019), which should also be concerned on maize next.

Under various environmental stress, compatible solutes are accumulated to protect plant cell from osmotic pressure and oxidative damage (Matysik et al. 2002; Cuin and Shabala 2007; Szabados and Savouré 2010; Gupta and Huang 2014). In this study, proline and soluble sugar contents were induced in tolerant QXN233 under Pi deficiency, except for the soluble protein content, implying an importance of proline and soluble sugar in maize response to LP stress, which could be in function as osmoprotectants to reduce the membrane damage and maintain ion balance. In addition, proline content was found to be associated with grain productivity in the maize (Spoljarević et al. 2011). Thus, it was inferred that the tolerant QXN233 had superior performance importantly related with its high proline content. Furthermore, proline accumulation is attributed to the enhanced transcript levels of proline-synthesis genes P5CR and P5CS in QXN233 plant. Sugars are required for the plant growth and development (Avonce et al. 2005; Elbein et al. 2003; Gupta and Kaur 2005), and also act as osmoprotectant or a signal molecule in signal transduction under different stresses (Yeo et al. 2000; Gupta and Kaur 2005). In this study, the tolerant QXN233 has higher soluble sugar content than MH05-4, consistent with its enhanced transcription of ZmTPS1, which could promote the strong growth under LP stress. Previously, SOD is considered as the most effective intracellular enzymatic protective system to scavenge excessive ROS under adverse stress (Gill and Tuteja 2010; Sun et al. 2016a, b). In this study, an increase of SOD activity was detected in QXN233 under Pi deficiency, in line with its remarkably high expression levels of $\mathrm{ZmSOD} 4$ and $\mathrm{ZmPOD3}$. 
A

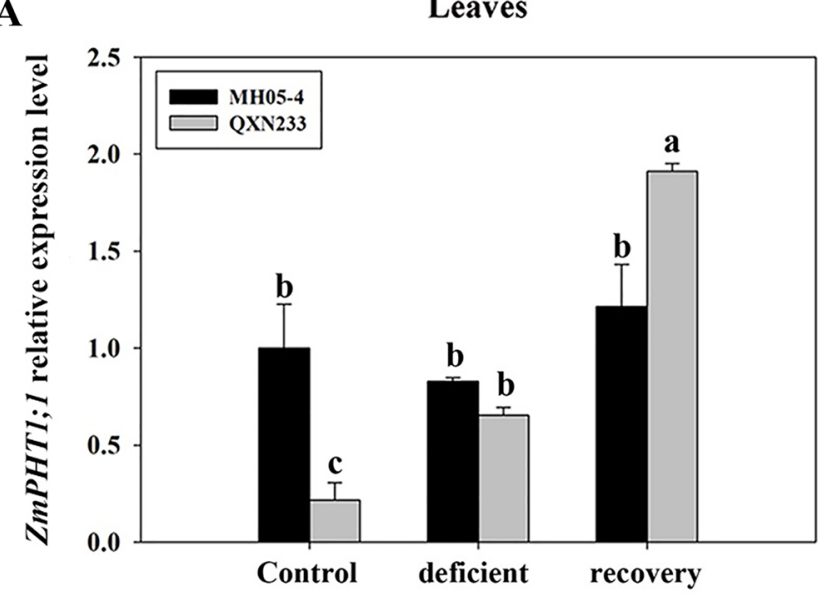

C

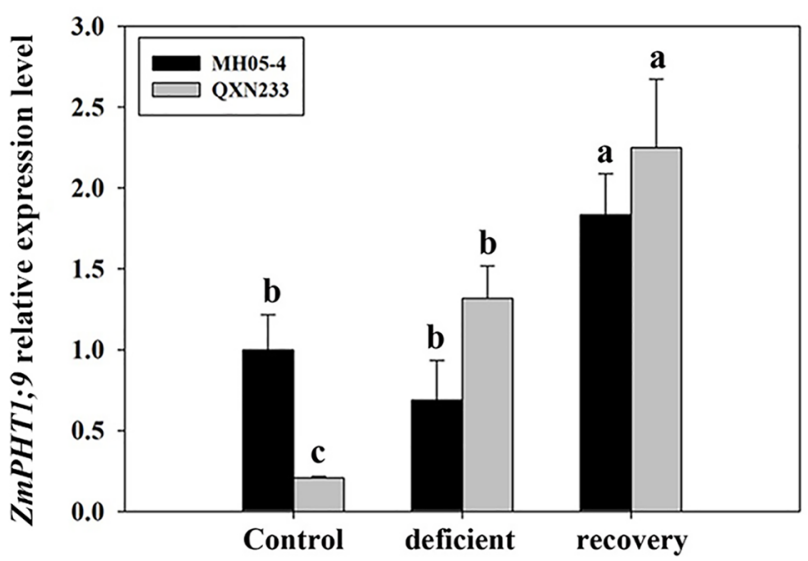

E

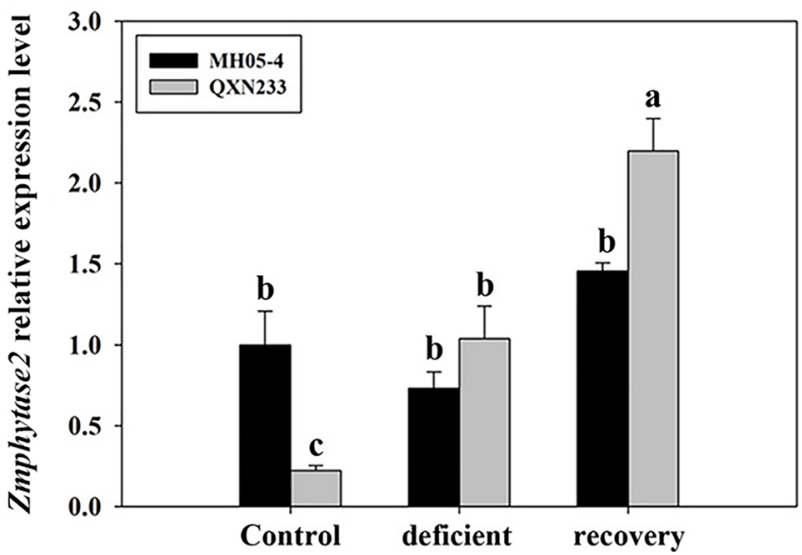

Fig. 5 Expression analysis of two Pi transporter genes and phytase in QXN233 and MH05-4 by qRT-PCR. The transcript levels of ZmPHT1;1 (A or B), ZmPHT1;9 (C or D), and Zmphytase (E or F)
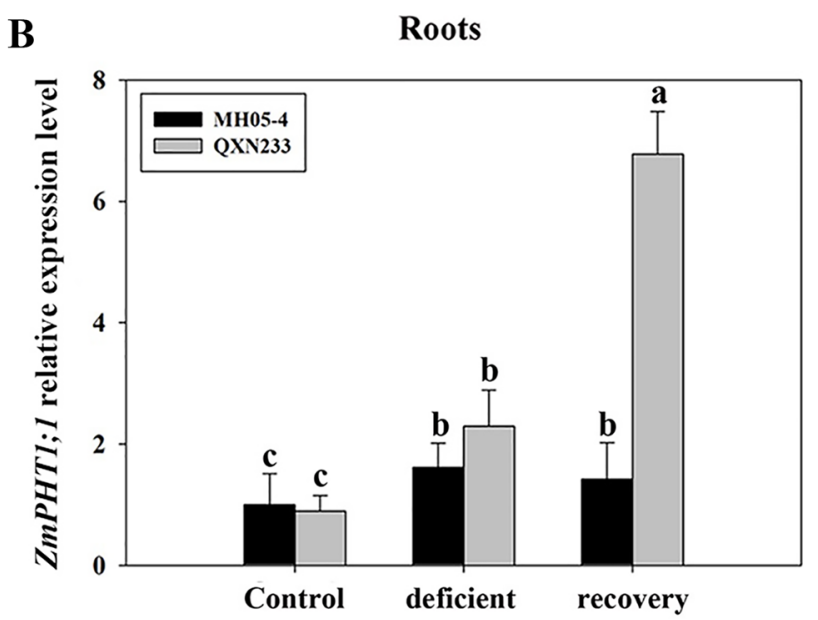

D

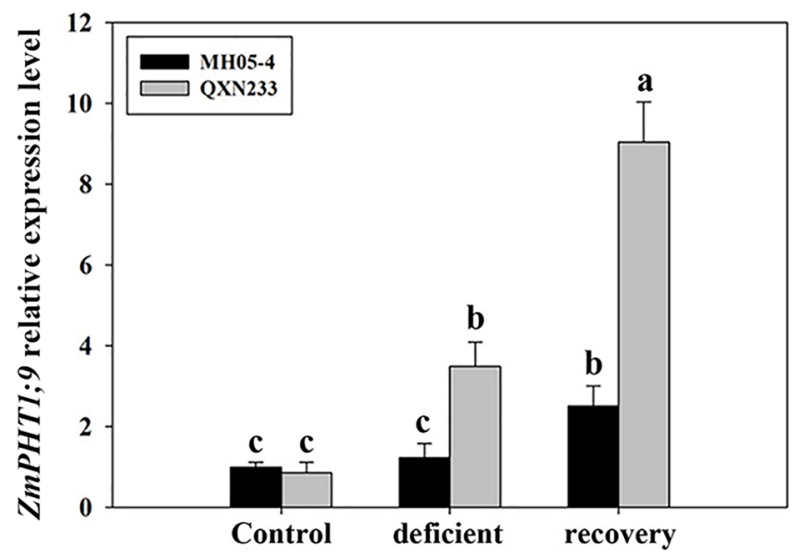

F

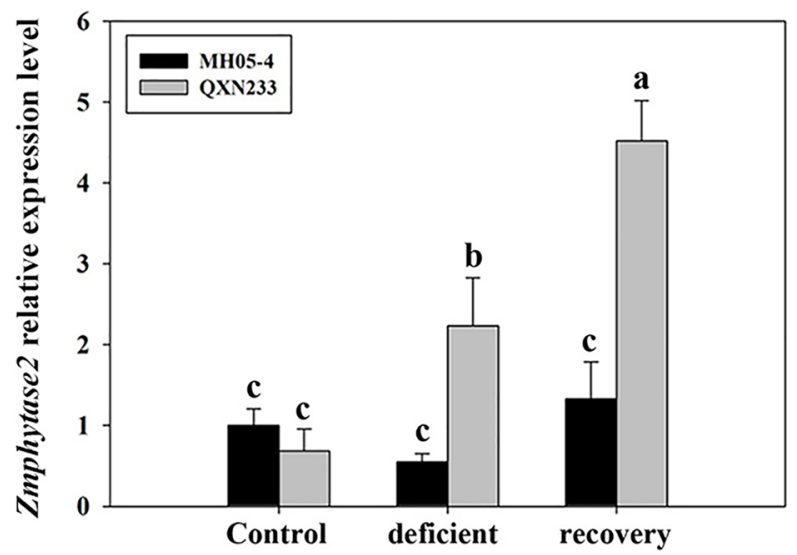

in leaves or roots. Maize seedlings were treated for 14 days under $\mathrm{Pi}$ deficiency and followed 2 days of Pi resupply. Values given as mean $\pm \mathrm{SD}(P<0.05, n=5$ biological replicates $)$ 
Fig. 6 Expression analysis of $\mathrm{ZmP5CR}$ (A), ZmP5CS (B), ZmTPS1 (C), ZmSOD4 (D), and ZmPOD3 (E) genes in QXN233 and MH05-4. Maize seedlings were treated for 2 days under Pi deficiency. Values given as mean $\pm \mathrm{SD}(P<0.05, n=5$ biological replicates)
A

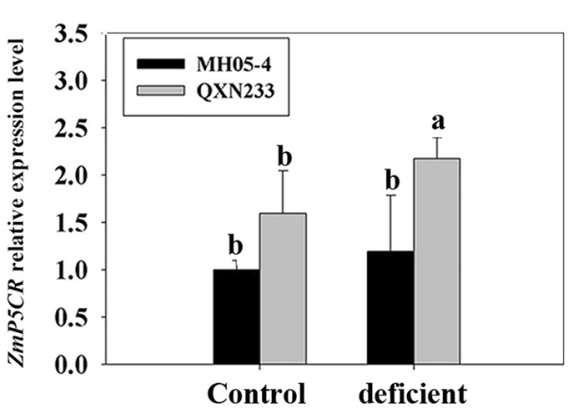

C

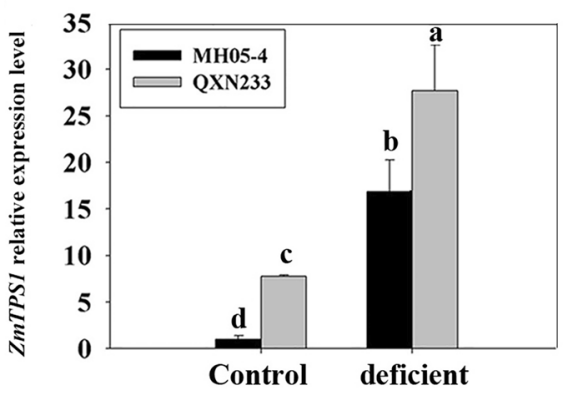

$\mathbf{E}$

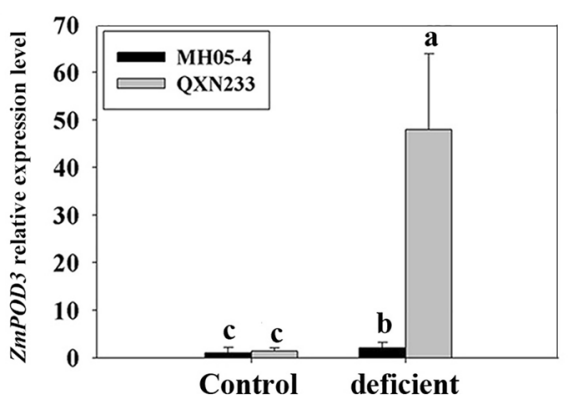

B

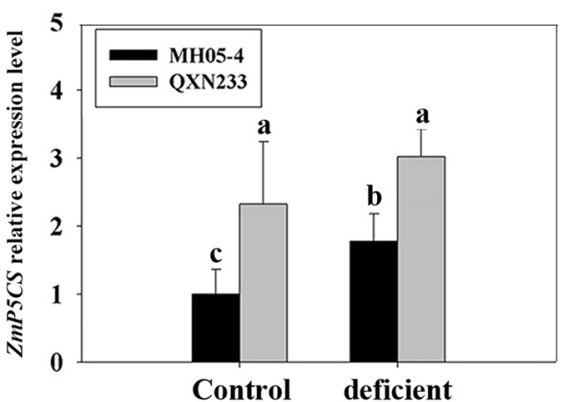

D

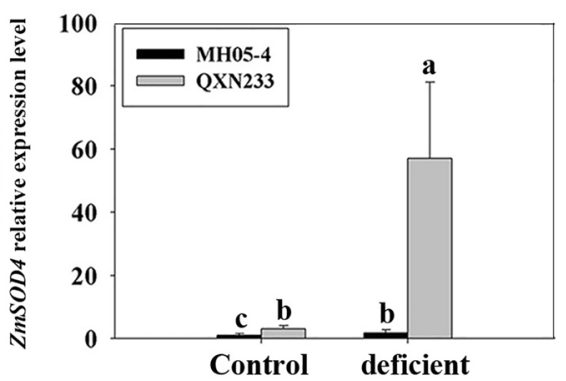

\section{Conclusions}

Two contrasting maize breeding lines, QXN233 and MH05-4, exhibit a tolerant and sensitive phenotype under Pi deficiency, respectively. QXN233 possessed high Pi content, compatible solutes, and SOD activity relative to MH05-4. Importantly, some Pi deficiency responsive genes, including ZmPHT1;1, ZmPHT1;9, and Zmphytase 2, play important roles for acquiring Pi in soil, especially for its roots under Pi recovery, indicated that these genes play vital roles in regulating $\mathrm{Pi}$ homeostasis under $\mathrm{Pi}$ deficiency.

Supplementary Information The online version contains supplementary material available at https://doi.org/10.1007/s11105-021-01310-w.

Acknowledgements The authors thank Professor Chunhua Mu for the seeds supply and Professor Xia Liu and Dr. Hua Zhang for the constructive suggestions.
Author contributions Statement Y.S. performed experiment and contributed reagents and materials. Y.S. and H.Z. contributed in write up.

Funding The work was supported by the Natural Science Foundation of Shandong Province (ZR2017BC021) and the National Natural Science Foundation of China (31902408).

\section{Declarations}

Conflict of Interest The authors declare no competing interests.

Open Access This article is licensed under a Creative Commons Attribution 4.0 International License, which permits use, sharing, adaptation, distribution and reproduction in any medium or format, as long as you give appropriate credit to the original author(s) and the source, provide a link to the Creative Commons licence, and indicate if changes were made. The images or other third party material in this article are included in the article's Creative Commons licence, unless indicated otherwise in a credit line to the material. If material is not included in the article's Creative Commons licence and your intended use is not permitted by statutory regulation or exceeds the permitted use, you will 
need to obtain permission directly from the copyright holder. To view a copy of this licence, visit http://creativecommons.org/licenses/by/4.0/.

\section{References}

Avonce N, Leyman B, Thevelein J, Iturriaga G (2005) Trehalose metabolism and glucose sensing in plants. Biochem Soc $\mathrm{T}$ 33:276-279

Calderon-Vázquez C, Ibarra-Laclette E, Caballero-Perez J, HerreraEstrella L (2008) Transcript profiling of Zea mays roots reveals gene responses to phosphate deficiency at the plant- and speciesspecific levels. J Exp Bot 59:2479-2497

Calderón-Vázquez C, Sawers RJ, Herrera-Estrella L (2011) Phosphate deprivation in maize: genetics and genomics. Plant Physiol 156:1067-1077

Ceasar SA, Ramakrishnan M, Vinod KK, Roch GV, Upadhyaya HD, Baker A, Ignacimuthu S (2020) Phenotypic responses of foxtail millet (Setaria italica) genotypes to phosphate supply under greenhouse and natural field conditions. PLoS One 15:e0233896

Chen JB, Yang JW, Zhang ZY, Feng XF, Wang SM (2013) Two P5CS genes from common bean exhibiting different tolerance to salt stress in transgenic Arabidopsis. J Genet 92:461-469

Chen R, Xue G, Chen P, Yao B, Yang W, Ma Q, Fan Y, Zhao Z, Tarczynski MC, Shi J (2008) Transgenic maize plants expressing a fungal phytase gene. Transgenic Res 17:633-643

Chiou TJ, Lin SI (2011) Signaling network in sensing phosphate availability in plants. Ann Rev Plant Biol 62:85-206

Cuin TA, Shabala S (2007) Compatible solutes reduce ROS-induced potassium efflux in Arabidopsis roots. Plant Cell Eviron 30:875-885

Elbein AD, PanYT PI, Carroll D (2003) New insights on trehalose: a multifunctional molecule. Glycobiology 13:17R-27R

Fang ZY, Shao C, Meng YJ, Wu P, Chen M (2009) Phosphate signaling in Arabidopsis and Oryza sativa. Plant Sci 176:170-180

Franco-Zorrilla JM, Valli A, Todesco M, Mateos I, Puga MI, RubioSomoza I, Leyva A, Weigel D, García JA, Paz-Ares J (2007) Target mimicry provides a new mechanism for regulation of microRNA activity. Nat Genet 39:1033-1037

Gill SS, Tuteja N (2010) Reactive oxygen species and antioxidant machinery in abiotic stress tolerance in crop plants. Plant Physiol Biochem 48:909-930

Gu M, Chen A, Sun S, Xu G (2016) Complex regulation of plant phosphate transporters and the gap between molecular mechanisms and practical application: what is missing? Mol Plant 9:396-416

Gupta AK, Kaur N (2005) Sugar signalling and gene expression in relation to carbohydrate metabolism under abiotic stresses in plants. 30:761-776

Gupta B, Huang B (2014) Mechanism of salinity tolerance in plants: physiological, biochemical, and molecular characterization. Int J Genomics 2014:701596

Ham BK, Chen J, Yan Y, Lucas WJ (2018) Insights into plant phosphate sensing and signaling. Curr Opin Biotechnol 49:1-9

Jiang H, Zhang J, Han Z, Yang J, Ge C, Wu Q (2017) Revealing new insights into different phosphorus-starving responses between two maize (Zea mays) inbred lines by transcriptomic and proteomic studies. Sci Rep 7:44294

Liang C, Wang J, Zhao J, Tian J, Liao H (2014) Control of phosphate homeostasis through gene regulation in crops. Curr Opin Plant Biol 21:59-66

Lin HJ, Gao J, Zhang ZM, Shen YO, Lan H, Liu L, Xiang K, Zhao M, Zhou S, Zhang YZ, Gao SB, Pan GT (2013) Transcriptional responses of maize seedling root to phosphorus starvation. Mol Biol Rep 40:5359-5379
Lin WY, Lin SI, Chiou TJ (2009) Molecular regulators of phosphate homeostasis in plants. J Exp Bot 60:1427-1438

Liu F, Xu Y, Jiang H, Jiang C, Du Y, Gong C, Wang W, Zhu S, Han G, Cheng B (2016) Systematic identification, evolution and expression analysis of the Zea mays PHT1 gene family reveals several new members involved in root colonization by arbuscular mycorrhizal fungi. Int J Mol Sci 17:930

Liu N, Shang W, Li C, Jia L, Wang X, Xing G, Zheng W (2018) Evolution of the SPX gene family in plants and its role in the response mechanism to phosphorus stress. Open Biol 8:170231

López-Arredondo DL, Leyva-González MA, González-Morales SI, López-Bucio J, Herrera-Estrella L (2014) Phosphate nutrition: improving low-phosphate tolerance in crops. Ann Rev Plant Biol 65:95-123

Matysik J, Bhalu BA, Mohanty P (2002) Molecular mechanisms of quenching of reactive oxygen species by proline under stress in plants. Curr Sci 82:525-532

Misson J, Thibaud MC, Bechtold N, Raghothama KG, Nussaume L (2004) Transcriptional regulation and functional properties of Arabidopsis $\mathrm{Pht} 1 ; 4$, a high affinity transporter contributing greatly to phosphate uptake in phosphate deprived plants. Plant Mol Biol $55: 727-741$

Mitra GN (2015) Phosphate (Pi) uptake. Regulation of Nutrient Uptake by Plants: A Biochemical and Molecular Approach. Springer India, India, pp 25-42

Mitsukawa N, Okumura S, Shirano Y, Sato S, Kato T, Harashima S, Shibata D (1997) Overexpression of an Arabidopsis thaliana high-affinity phosphate transporter gene in tobacco cultured cells enhances cell growth under phosphate-limited conditions. Proc Natl Acad Sci USA 94:7098-7102

Mudge SR, Rae AL, Diatloff E, Smith FW (2002) Expression analysis suggests novel roles for members of the Pht 1 family of phosphate transporters in Arabidopsis. Plant J 31:341-353

Müller J, Toev T, Heisters M, Teller J, Moore KL, Hause G, Dinesh DC, Bürstenbinder K, Abel S (2015) Iron-dependent callose deposition adjusts root meristem maintenance to phosphate availability. Dev Cell 33:216-230

Nagy R, Vasconcelos MJV, Zhao S, McElver J, Bruce W, Amrhein N, Raghothama KG, Bucher M (2006) Differential regulation of five Pht1 phosphate transporters from maize (Zea mays L.). Plant Biol 8:186-197

Nuccio ML, Rhodes D, McNeil SD, Hanson AD (1999) Metabolic engineering of plants for osmotic stress resistance. Curr Opin Biotechnol 2:128-134

Oono Y, Kobayashi F, Kawahara Y, Yazawa T, Handa H, Itoh T, Matsumoto T (2013) Characterization of the wheat (Triticum aestivum L.) transcriptome by de novo assembly for the discovery of phosphate starvation-responsive genes: gene expression in $\mathrm{Pi}$ stressed wheat. BMC genomics 14:77

Pei LM, Jin Z, Li KP, Yin HY, Wang JM, Yang AF (2013) Identification and comparative analysis of low phosphate toleranceassociated microRNAs in two maize breeding lines. Plant Physiol Biochem 70:221-234

Poirier Y, Bucher M (2002) Phosphate transport and homeostasis in Arabidopsis. In: Somerville CR, Meyerowitz EM, Rockville MD (ed) The Arabidopsis book. The American Society of Plant Biologists, 15501 Monona Drive Rockville, MD 20855, USA, pp 1-35

Rai AN, Penna S (2013) Molecular evolution of plant P5CS gene involved in proline biosynthesis. Mol Biol Rep 40:6429-6435

Rausch C, Bucher M (2002) Molecular mechanisms of phosphate transport in plants. Planta 216:23-37

Ried MK, Wild R, Zhu J, Pipercevic J, Sturm K, Broger L, Harmel RK, Abriata LA, Hothorn LA, Fiedler D, Hiller S, Hothorn M (2021) Inositol pyrophosphates promote the interaction of SPX domains with the coiled-coil motif of PHR transcription factors to regulate plant phosphate homeostasis. Nat Commun 12:384 
Ruan W, Guo M, Xu L, Wang X, Zhao H, Wang J, Yi K (2018) An SPX-RLI1 module regulates leaf inclination in response to phosphate availability in rice. Plant Cell 30:853-870

Rubio V, Linhares F, Solano R, Martín AC, Iglesias J, Leyva A, PazAres J (2001) A conserved MYB transcription factor involved in phosphate starvation signaling both in vascular plants and in unicellular algae. Genes Dev 15:2122-2133

Sawers RJ, Svane SF, Quan C, Grønlund M, Wozniak B, Gebreselassie MN, González-González-Muñoz E, Chávez Montes RA, Baxter I, Goudet J, Jakobsen I, Paszkowski U (2017) Phosphorus acquisition efficiency in arbuscular mycorrhizal maize is correlated with the abundance of root-external hyphae and the accumulation of transcripts encoding PHT1 phosphate transporters. New Phytol 214:632-643

Secco D, Wang C, Arpat BA, Wang Z, Poirier Y, Tyerman SD, Wu P, Shou H, Whelan J (2012) The emerging importance of the SPX domain-containing proteins in phosphate homeostasis. New Phytol 193:842-851

Schachtman DP, Reid RJ, Ayling SM (1998) Phosphorus uptake by plants: from soil to cell. Plant Physiol 116:447-453

Schmittgen TD, Livak KJ (2008) Analyzing real-time PCR data by the comparative CT method. Nat Protoc 3:1101-1108

Shin H, Shin HS, Dewbre GR, Harrison MJ (2004) Phosphate transport in Arabidopsis: Pht1;1 and Pht1;4 play a major role in phosphate acquisition from both low- and high-phosphate environments. Plant J 39:629-642

Spoljarević M, Agić D, Lisjak M, Gumze A, Wilson ID, Hancock JT, Teklić T (2011) The relationship of proline content and metabolism on the productivity of maize plants. Plant Signal Behav 6:251-257

Stefanovic A, Ribot C, Rouached H, Wang Y, Chong J, Belbahri L, Delessert S, Poirier Y (2007) Members of the PHO1 gene family show limited functional redundancy in phosphate transfer to the shoot, and are regulated by phosphate deficiency via distinct pathways. Plant J 50:982-994

Sun Y, Kong X, Li C, Liu Y, Ding Z (2015) Potassium retention under salt stress is associated with natural variation in salinity tolerance among Arabidopsis accessions. PLoS One 10:e0124032

Sun Y, Mu C, Chen Y, Kong X, Xu Y, Zheng H, Zhang H, Wang Q, Xue Y, Li Z, Ding Z, Liu X (2016a) Comparative transcript profiling of maize inbreds in response to long-term phosphorus deficiency stress. Plant Physiol Biochem 109:467-481

Sun T, Li M, Shao Y, Yu L, Ma F (2016b) Comprehensive genomic identification and expression analysis of the phosphate transporter $(P H T)$ gene family in apple. Front Plant Sci 8:426
Sunarpi Horie T, Motoda J, Kubo M, Yang H, Yoda K, Horie R, Chan WY, Leung HY, Hattori K, Konomi M, Osumi M, Yamagami M, Schroeder JI, Uozumi N (2005) Enhanced salt tolerance mediated by AtHKT1 transporter-induced $\mathrm{Na}^{+}$unloading from xylem vessels to xylem parenchyma cells. Plant J 44:928-938

Szabados L, Savouré A (2010) Proline: a multifunctional amino acid. Trends Plant Sci 15:89-97

Vance CP, Uhde-Stone C, Allan DL (2003) Phosphorus acquisition and use: critical adaptations by plants for securing a nonrenewable resource. New Phytol 157:423-447

Verslues PE, Sharma S (2010) Proline metabolism and its implications for plant environment interaction. Arabidopsis Book 8:e0140

Vinocur B, Altman A (2005) Recent advances in engineering plant tolerance to abiotic stress: achievements and limitations. Curr Opin Biotechnol 16:123-132

Wang D, Lv S, Jiang P, Li Y (2017) Roles, regulation, and agricultural application of plant phosphate transporters. Front Plant Sci 8:817

Wang X, Wang Z, Zheng Z, Dong J, Song L, Sui L, Nussaume L, Desnos T, Liu D (2019) Genetic dissection of Fe-dependent signaling in root developmental responses to phosphate deficiency. Plant Physiol 179:300-316

Wang Y, Ribot C, Rezzonico E, Poirier Y (2004) Structure and expression profile of the Arabidopsis $\mathrm{PHOl}$ gene family indicates a broad role in inorganic phosphate homeostasis. Plant Physiol 135:400-411

Xu L, Zhao H, Wan R, Liu Y, Xu Z, Tian W, Ruan W, Wang F, Deng M, Wang J, Dolan L, Luan S, Xue S, Yi K (2019) Identification of vacuolar phosphate efflux transporters in land plants. Nat Plants 5:84-94

Xu Y, Liu F, Han G, Cheng B (2018) Genome-wide identification and comparative analysis of phosphate starvation-responsive transcription factors in maize and three other gramineous plants. Plant Cell Rep 37:711-726

Yang XJ, Finnegan PM (2010) Regulation of phosphate starvation responses in higher plants. Ann Bot 105:513-526

Yeo ET, Kwon HB, Han SE, Lee JT, Ryu JC, Byu MO (2000) Genetic engineering of drought resistant potato plants by introduction of the trehalose-6-phosphate synthase (TPS1) gene from Saccharomyces cerevisiae. Mol Cells 10:263-268

Zhang X, Wang B, Zhao Y, Zhang J, Li Z (2019) Auxin and GA signaling play important roles in the maize response to phosphate deficiency. Plant Sci 283:177-188

Publisher's Note Springer Nature remains neutral with regard to jurisdictional claims in published maps and institutional affiliations. 